\title{
Variabilidade espacial das propriedades físicas e químicas do solo em áreas intensamente cultivadas ${ }^{1}$
}

\author{
Gláucia de Mello², Célia R. P. Bueno² \& Gener T. Pereira ${ }^{2}$
}

\begin{abstract}
RESUMO
Neste trabalho, avaliou-se a variabilidade espacial das propriedades físicas e químicas do solo, visando fornecer subsídios ao manejo localizado de insumos. Foram analisadas as variáveis químicas: $\mathrm{P}, \mathrm{MO}, \mathrm{K}, \mathrm{Ca}, \mathrm{Mg}$, $\mathrm{pH}, \mathrm{CTC}$ e $\mathrm{V}(\%)$ e físicas: areia e argila. Coletaram-se amostras de solo em duas profundidades (0-0,2 e 0,6-0,8 m) situadas em malha irregular de amostragem na região de Monte Alto, num Argissolo Vermelho-Amarelo (PVA), sob diferentes manejos, perfazendo 88 pontos em área total de 1465 ha; e na região de Jaboticabal, em Latossolo Vermelho (LV) cultivado com cana-de-açúcar, perfazendo 128 pontos, em área total de 2597 ha. As propriedades químicas e físicas dos solos estudados apresentaram dependência espacial, com exceção da CTC na profundidade de 0,6-0,8 m para o solo LV; Ca e argila na profundidade de 0-0,2 m, e P, MO, K, Mg e argila na camada de 0,6-0,8 m, no solo PVA. As variáveis estudadas ajustaram-se aos modelos esférico e exponencial, e algumas apresentaram semivariograma sem estrutura definida. O solo PVA apresentou menor continuidade espacial das propriedades químicas e físicas, principalmente na profundidade 0,6-0,8 m, camada que sofre menor influência antrópica. O solo LV apresentou zonas mais homogêneas de fertilidade e granulometria.
\end{abstract}

Palavras-chave: geoestatística, fertilidade do solo, granulometria

\section{Spatial variability of physical and chemical properties of soil in intensively cultivated areas}

\begin{abstract}
The spatial variability of physical and chemical properties of soil were evaluated to provide subsidies for management of the agricultural input. The chemical variables: $\mathrm{P}$, organic matter (OM), K, $\mathrm{Ca}, \mathrm{Mg}, \mathrm{pH}, \mathrm{CEC}$ and base saturation (BS); and physical variables: sand and clay were analysed. Soil samples were collected at two depths (0-0.2 and 0.6-0.8 m) located at irregular mesh of sampling in the region of Monte Alto, in a Yellow-Red Podzol (Alfissolo) (PVA), under different managements, resulting in 88 points in 1465 ha of total area; and at the region of Jaboticabal in a Red Latosol (LV) cultivated with sugarcane, resulting in 128 points in 2597 ha of total area. The chemical and physical soil properties studied showed spatial dependence, except CEC in 0.6-0.8 m layer for LV; Ca and clay at 0-0.2 m layer and P, OM, K, $\mathrm{Mg}$, sand and clay in 0.6-0.8 m layer for PVA. The chemical and physical variables studied were adjusted to spherical and exponential models and some of them showed semivariogram without defined structure. The PVA showed low spatial continuity of chemical and physical properties, mainly in 0.6-0.8 m layer, which suffers less antropic influence. The LV soil showed more homogenous zones of fertility and granulometric composition.
\end{abstract}

Key words: geostatistic, soil fertility, granulometric composition

1 Parte da Dissertação de Mestrado do primeiro autor, apresentada à UNESP, Campus de Jaboticabal, SP, financiada com bolsa CNPq ${ }^{2}$ FCAV/UNESP, CEP 14884-900, Jaboticabal, SP, Fone: (64) 34913082. E-mail: glaucia_mello@ig.com.br, Fone: (16) 32092672, E-mail:crbueno@fcav.unesp.br, Fone: (16) 32092624, E-mail:genertp@fcav.unesp.br 


\section{INTRODUÇÃO}

Para um correto planejamento conservacionista de qualquer área de interesse, são necessários estudos detalhados que proporcionam sucesso e se possa usar os recursos naturais sem agredir, de forma degradante, o meio ambiente.

O conhecimento das propriedades físicas dos solos envolvidos, principalmente das relacionadas à distribuição granulométrica, que influenciam diretamente o fluxo superficial e o movimento de água no solo, são fundamentais para o planejamento ambiental.

É importante, também, o estudo do nível de fertilidade, pois muitas vezes o fator limitante do rendimento das culturas é o baixo teor de nutrientes, considerando-se que o maior rendimento aumenta a quantidade de material vegetal que fica como cobertura do solo, sendo este um dos fatores controladores da erosão. Constata-se, assim uma relação entre fertilidade e erosão, podendo levar à diminuição do arraste de partículas devido ao aumento de fertilidade, e redução da degradação progressiva do solo e, quando a fertilidade é baixa, ocasiona maiores perdas por erosão, reduzindo a quantidade de nutrientes disponíveis às plantas, tornando o solo mais pobre.

Por outro lado, a produção de cobertura vegetal afeta o teor de matéria orgânica do solo, que é muito importante para a sua conservação, à medida que, quanto maior o teor de matéria orgânica, maior também a estabilidade dos agregados, melhorando a estrutura do solo, de forma a permitir maior infiltração e diminuir o escoamento superficial de água.

A variabilidade espacial dos atributos físicos, químicos e biológicos do solo, influencia a eficiência do manejo das culturas e seu desenvolvimento.

Além da variabilidade natural do solo, as práticas agrícolas de manejo são fontes adicionais de variabilidade (Souza et al.,1997). O manejo do solo pode afetar propriedades químicas, físicas, mineralógicas e biológicas, com impacto principalmente nas camadas superficiais (Beckett \& Webster, 1971). O conhecimento desta variação é imprescindível para o levantamento e classificação de solos, para a avaliação de sua fertilidade, para o desenvolvimento de esquemas de amostragens mais adequados, para o planejamento experimental e para as definições de práticas apropriadas de manejo e recuperação (Souza, 1992).

Para o estudo da dependência espacial de variáveis, utiliza-se a geoestatística, sendo a análise exploratória dos dados uma etapa de fundamental importância e obrigatória dentro de qualquer estudo desse tipo (Burrough et al., 1996). O estudo da variabilidade espacial é necessário em áreas cujo solo está submetido a diferentes práticas culturais, indicando alternativas não só para reduzir os efeitos da variabilidade do solo na produção de culturas (Trangmar et al., 1985) mas, também, para estimar respostas das propriedades do solo em função de determinadas práticas de manejo (Ovalles \& Rey, 1994).

As técnicas geoestatísticas, com base na construção de semivariogramas, permitem a descrição da dependência espacial das propriedades dos solos (Vieira, 2000). Vários au- tores têm estudado a variabilidade espacial de propriedades químicas e físicas dos solos. Souza et al. (1997) estudando a variabilidade espacial em Latossolo Amarelo distrófico na profundidade de $0-0,2 \mathrm{~m}$, em uma transeção composta por uma única linha de 50 plantas espaçadas $4 \mathrm{~m}$, em um pomar cítrico; encontraram modelos dos semivariogramas ajustados ao esférico para as variáveis $\mathrm{pH}, \mathrm{Ca}, \mathrm{Mg}$ e argila; exponencial para as variáveis CTC e areia; gaussiano para a variável SB, e V(\%) não apresentou estrutura definida. No seu trabalho, Salviano et al. (1998) em Argissolo Vermelho-Amarelo, com distância entre pontos de $5 \mathrm{~m}$, encontraram dependência espacial com ajuste ao modelo esférico nas profundidades de $0-0,2$ e 0,2-0,4 m para as variáveis $\mathrm{K}, \mathrm{Ca}, \mathrm{Mg}, \mathrm{pH}, \mathrm{CTC}, \mathrm{V}(\%), \mathrm{MO}$ e argila, e para a variável areia na profundidade de $0,2-0,4 \mathrm{~m}$; que na camada de 0-0,2 m se ajustou ao modelo gaussiano; a variável $\mathrm{P}$ na profundidade de $0-0,2 \mathrm{~m}$ ajustou-se ao modelo esférico, que na profundidade de 0,2-0,4 m não apresentou dependência espacial. Resultados semelhantes foram encontrados por Oliveira et al. (1999) na profundidade de 0-0,3 m para as variáveis K, Ca e CTC, exceção para o $\mathrm{pH}$ e $\mathrm{Mg}$, que se ajustaram melhor ao modelo gaussiano e linear, respectivamente.

Objetivou-se, com este trabalho, avaliar a variabilidade espacial das propriedades físicas e químicas em duas regiões com diferentes: material de origem, relevo classe de solo e cultivo, procurando-se fornecer subsídios ao manejo localizado de insumos.

\section{MATERIAL E MÉTODOS}

O estudo foi realizado em duas áreas distintas: uma localizada no município de Monte Alto, SP, entre longitude de $48^{\circ} 26^{\prime}$ e $48^{\circ} 29^{\prime} \mathrm{W}$ e latitude de $21^{\circ} 15^{\prime}$ e $21^{\circ} 19^{\prime} \mathrm{S}$, com altitude média de $650 \mathrm{~m}$ e área de 1465 ha; e a outra no município de Jaboticabal, SP, entre longutide $48^{\circ} 10^{\prime}$ e $48^{\circ} 15^{\prime} \mathrm{W}$ e latitude $21^{\circ} 11^{\prime}$ e $21^{\circ} 14^{\prime} \mathrm{S}$, altitude média de 550 m, com área de 2597 ha (Figura 1). O tipo de solo predominante na área de estudo em Monte Alto é o Argissolo Vermelho-Amarelo (PVA) eutrófico textura arenosa a média, relevo ondulado, desenvolvidos dos arenitos do Grupo Bauru - Formação Marília, inserido no Planalto Ocidental Paulista; na área em Jaboticabal predomina Latossolo Vermelho (LV) eutroférrico textura argilosa a muito argilosa, relevo suave ondulado, desenvolvido dos basaltos do Grupo São Bento-Formação Serra Geral, inserido nas Cuestas Basálticas.

O clima da região, de acordo com a classificação de Köppen, é do tipo mesotérmico úmido subtropical de inverno seco (Cwa), com precipitação total de 1400 mm e temperatura média variando de 21,5 a $24,7^{\circ} \mathrm{C}$; a vegetação natural original é do tipo floresta latifoliada tropical. A área de Monte Alto é cultivada com frutíferas, pastagens e cana-deaçúcar, enquanto a de Jaboticabal é totalmente cultivada com cana-de-açúcar.

Coletaram-se amostras de solos georreferenciadas, em grade irregular, em 88 pontos na área com PVA e 128 pontos na 


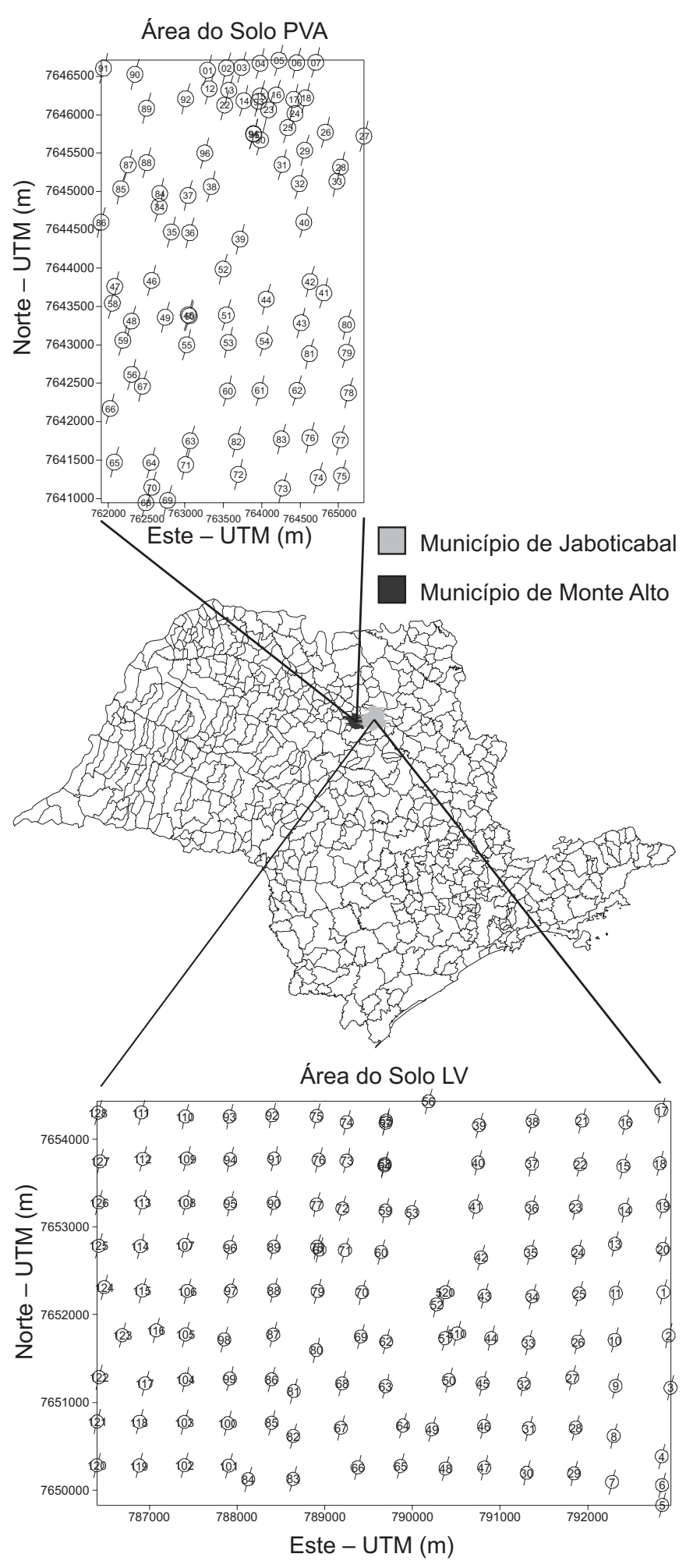

Figura 1. Localização das áreas de estudo, com representação da malha de amostragem do solo LV no município de Jaboticabal, SP, e do solo PVA, no município de Monte Alto, SP

área com LV, distanciados em média $500 \mathrm{~m}$, nas profundidades de 0-0,2 e de 0,6-0,8 m. A análise textural foi realizada de acordo com o método proposto por Day (1965) modificado. A composição granulométrica da terra fina foi determinada com dispersão em água com $\mathrm{NaOH}$, agitação lenta (16 h), sedimentação, sendo a argila determinada pelo método da pipeta e a areia foi fracionada por tamisação. Para determinação das variáveis químicas, utilizou-se o método de van Raij et al. (1987).

As análises estatísticas descritivas foram realizadas pelo programa SAS (Schlotzhauer \& Littell, 1997). A análise da dependência espacial foi feita através do ajuste dos dados ao semivariograma experimental, de acordo com a teoria das variáveis regionalizadas, utilizando-se o programa $\mathrm{GS}^{+}$ v. 5.03 Beta (Robertson, 1998). Utilizaram-se lags com intervalos não uniformes para os ajustes aos modelos. Realizou-se a seleção dos modelos com base no melhor $\mathrm{R}^{2}$ (coeficiente de determinação) e menor SQR (soma de quadrados reduzida). Após a obtenção dos gráficos de ajuste do modelo do semivariograma, fez-se a interpolação de dados (Krigagem), com vistas a definir o padrão espacial das variáveis estudadas, e exportados os arquivos para o programa SURFER v. 6.01 (Golden Software, 1995).

\section{RESULTADOS E DISCUSSÃO}

Os resultados das análises estatísticas descritivas encontram-se na Tabela 1. Ocorreram valores com certo distanciamento entre a média e a mediana para os dois solos, o que pode ser um indicativo de distribuição de dados assimétricos, em que as medidas de tendência central são dominadas por valores atípicos. Isto é mais acentuado para algumas variáveis, tais como os valores máximos e mínimos das variáveis areia e P nas duas profundidades nos solos LV e PVA, observando-se o mesmo padrão de comportamento para as demais variáveis nos dois solos. Esses resultados concordam com os obtidos por Araújo (2002) em Latossolo Vermelho, na região de Jaboticabal cultivado também com cana-de-açúcar. Esta grande amplitude de valores observados revela os problemas que podem ocorrer quando se usa a média das variáveis para a realização do manejo do solo, ou seja, quando a análise de solo, da maneira como é feita atualmente, usando-se a amostra composta, para recomendar adubação, podendo haver aplicação excessiva em alguns locais e insuficientes em outros, causando prejuízos econômicos.

Na profundidade de 0,6-0,8 m, os valores médios, medianos, máximos e mínimos das propriedades químicas, foram menores que na profundidade $0-0,2 \mathrm{~m}$ nos dois solos, com exceção do $\mathrm{pH}$ do solo LV. Os valores mais elevados na camada superficial deste solo são devidos, provavelmente, ao maior teor de matéria orgânica e CTC, e às constantes aplicações de fertilizantes e corretivos. Valores de $\mathrm{pH}$ mais baixos na camada superficial do solo LV, também foram encontrados por outros autores (Pocay, 2000; Souza, 2001; Araújo, 2002), que apresentaram várias possíveis explicações para este comportamento, tais como as condições específicas de manejo da cultura da cana-de-açúcar, que promovem a extração elevada de nutrientes, principalmente cálcio e magnésio, exaurindo o solo na camada superficial pelos anos sucessivos de cultivo, bem como o uso de adubação nitrogenada.

Para as propriedades físicas observa-se, em ambas as áreas, que os teores de areia são maiores na camada superficial (0-0,2 m), e de argila na camada de 0,6-0,8 m, evidenciando as variações de granulometria em profundidade, com 
Tabela 1. Valores da estatística descritiva das variáveis químicas e físicas dos solos Latossolo Vermelho (LV) e Argissolo Vermelho-Amarelo (PVA)

\begin{tabular}{|c|c|c|c|c|c|c|c|c|}
\hline Variáveis & Media & Mediana & Max. & Mín. & Desvio Padrão & Assimetria & Curtose & CV(\%) \\
\hline \multicolumn{9}{|c|}{ SOLO LV $\quad 0-0,2 \mathrm{~m}$} \\
\hline$P\left(\mathrm{mg} \mathrm{dm}^{-3}\right)$ & 22,7 & 18,00 & 112,00 & 4,00 & 16,73 & 2,17 & 6,84 & 74 \\
\hline $\mathrm{M} 0\left(\mathrm{~g} \mathrm{dm}^{-3}\right)$ & 28,54 & 28,00 & 42,00 & 15,00 & 5,17 & $-0,04$ & $-0,05$ & 18 \\
\hline $\mathrm{pH}$ em $\mathrm{CaCl}_{2}$ & 5,29 & 5,30 & 7,20 & 4,20 & 0,41 & 0,64 & 3,08 & 8 \\
\hline $\mathrm{K}\left(\mathrm{mmol}_{\mathrm{c}} \mathrm{dm}^{-3}\right)$ & 2,71 & 1,60 & 11,50 & 0,20 & 2,51 & 1,68 & 2,66 & 92 \\
\hline $\mathrm{Ca}\left(\mathrm{mmol}_{\mathrm{c}} \mathrm{dm}^{-3}\right)$ & 39,29 & 35,00 & 170,00 & 6,00 & 18,67 & 3,08 & 19,04 & 49 \\
\hline $\mathrm{Mg}\left(\mathrm{mmol}_{\mathrm{c}} \mathrm{dm}^{-3}\right)$ & 14,12 & 13,00 & 60,00 & 2,00 & 8,29 & 2,18 & 7,82 & 59 \\
\hline CTC & 92,00 & 89,60 & 242,90 & 50,70 & 21,33 & 2,95 & 18,82 & 23 \\
\hline V(\%) & 57,94 & 58,00 & 96,00 & 14,00 & 14,15 & $-0,29$ & 0,20 & 24 \\
\hline Areia $\left(\mathrm{g} \mathrm{kg}^{-1}\right)$ & 318 & 273 & 672 & 134 & 123,08 & 1,08 & 0,37 & 38 \\
\hline Argila $\left(\mathrm{g} \mathrm{kg}^{-1}\right)$ & 495 & 512 & 610 & 197 & 80,78 & $-1,25$ & 1,57 & 16 \\
\hline \multicolumn{9}{|c|}{ SOLO LV $\quad 0,6-0,8 \mathrm{~m}$} \\
\hline$P\left(\mathrm{mg} \mathrm{dm}^{-3}\right)$ & 5,03 & 4,00 & 17,00 & 1,00 & 3,49 & 1,34 & 1,56 & 69 \\
\hline $\mathrm{MO}\left(\mathrm{g} \mathrm{dm}^{-3}\right)$ & 14,69 & 14,00 & 40,00 & 6,00 & 4,70 & 2,05 & 8,70 & 32 \\
\hline $\mathrm{pH}$ em $\mathrm{CaCl}_{2}$ & 5,44 & 5,60 & 6,30 & 4,00 & 0,53 & $-0,69$ & $-0,25$ & 10 \\
\hline $\mathrm{K}\left(\mathrm{mmol}_{\mathrm{c}} \mathrm{dm}^{-3}\right)$ & 1,01 & 0,60 & 5,80 & 0,20 & 0,99 & 2,14 & 5,12 & 97 \\
\hline $\mathrm{Ca}\left(\mathrm{mmol}_{\mathrm{c}} \mathrm{dm}^{-3}\right)$ & 21,61 & 20,00 & 69,00 & 3,00 & 11,82 & 1,24 & 2,29 & 54 \\
\hline $\mathrm{Mg}\left(\mathrm{mmol}_{\mathrm{c}} \mathrm{dm}^{-3}\right)$ & 6,91 & 6,00 & 26,00 & 1,00 & 4,26 & 1,70 & 3,63 & 61 \\
\hline CTC & 55,15 & 49,75 & 191,20 & 33,40 & 19,89 & 3,53 & 18,66 & 36 \\
\hline V(\%) & 52,70 & 56,00 & 83,00 & 6,00 & 16,16 & $-0,53$ & $-0,18$ & 30 \\
\hline Areia $\left(\mathrm{g} \mathrm{kg}^{-1}\right)$ & 280 & 247 & 589 & 76 & 107,55 & 1,12 & 0,77 & 38 \\
\hline Argila $\left(\mathrm{g} \mathrm{kg}^{-1}\right)$ & 554 & 574 & 693 & 305 & 77,96 & $-1,06$ & 0,84 & 14 \\
\hline \multicolumn{9}{|c|}{ SOLO PVA $\quad 0-0,2 \mathrm{~m}$} \\
\hline$P\left(\mathrm{mg} \mathrm{dm}^{-3}\right)$ & 11,87 & 7,00 & 62,00 & 2,00 & 11,64 & 2,33 & 6,54 & 95 \\
\hline $\mathrm{M} 0\left(\mathrm{~g} \mathrm{dm}^{-3}\right)$ & 12,36 & 11,00 & 27,00 & 4,00 & 4,26 & 1,26 & 2,11 & 34 \\
\hline pH em $\mathrm{CaCl}_{2}$ & 5,17 & 5,10 & 6,50 & 4,10 & 0,60 & 0,23 & $-0,84$ & 11 \\
\hline $\mathrm{K}\left(\mathrm{mmol}_{\mathrm{c}} \mathrm{dm}^{-3}\right)$ & 2,65 & 2,10 & 17,50 & 0,60 & 2,36 & 4,64 & 25,83 & 89 \\
\hline $\mathrm{Ca}\left(\mathrm{mmol}_{\mathrm{c}} \mathrm{dm}^{-3}\right)$ & 19,70 & 18,00 & 53,00 & 4,00 & 9,77 & 0,71 & 0,51 & 49 \\
\hline $\mathrm{Mg}\left(\mathrm{mmol}_{\mathrm{c}} \mathrm{dm}^{-3}\right)$ & 8,09 & 7,00 & 25,00 & 1,00 & 4,45 & 1,14 & 2,01 & 55 \\
\hline CTC & 53,34 & 51,60 & 107,50 & 21,30 & 12,98 & 0,84 & 2,75 & 24 \\
\hline V(\%) & 56,18 & 59,00 & 83,00 & 12,00 & 17,90 & $-0,60$ & $-0,33$ & 32 \\
\hline Areia $\left(\mathrm{g} \mathrm{kg}^{-1}\right)$ & 787 & 789 & 923 & 623 & 56,3 & $-0,67$ & 0,78 & 7 \\
\hline Argila $\left(\mathrm{g} \mathrm{kg}^{-1}\right)$ & 159 & 153 & 326 & 72 & 49,1 & 0,88 & 1,05 & 31 \\
\hline \multicolumn{9}{|c|}{ SOLO PVA $\quad 0,6-0,8 \mathrm{~m}$} \\
\hline$P\left(\mathrm{mg} \mathrm{dm}^{-3}\right)$ & 2,26 & 2,00 & 10,00 & 1,30 & 1,30 & 3,02 & 14,42 & 57 \\
\hline MO $\left(\mathrm{g} \mathrm{dm}^{-3}\right)$ & 7,65 & 7,00 & 16,00 & 2,04 & 2,04 & 1,20 & 3,18 & 27 \\
\hline $\mathrm{pH}$ em $\mathrm{CaCl}_{2}$ & 4,79 & 4,70 & 5,90 & 0,49 & 0,49 & 0,47 & $-0,76$ & 10 \\
\hline $\mathrm{K}\left(\mathrm{mmol}_{\mathrm{c}} \mathrm{dm}^{-3}\right)$ & 1,27 & 1,00 & 4,80 & 0,84 & 0,84 & 2,00 & 5,36 & 66 \\
\hline $\mathrm{Ca}\left(\mathrm{mmol}_{\mathrm{c}} \mathrm{dm}^{-3}\right)$ & 18,76 & 17,00 & 47,00 & 11,09 & 11,09 & 0,70 & $-0,16$ & 59 \\
\hline $\mathrm{Mg}\left(\mathrm{mmol}_{\mathrm{c}} \mathrm{dm}^{-3}\right)$ & 5,57 & 5,00 & 12,00 & 2,55 & 2,55 & 0,76 & $-0,01$ & 46 \\
\hline CTC & 59,31 & 53,10 & 292,80 & 32,74 & 32,74 & 5,47 & 35,04 & 55 \\
\hline V(\%) & 45,45 & 48,00 & 76,00 & 17,73 & 17,73 & $-0,46$ & $-0,41$ & 39 \\
\hline Areia $\left(\mathrm{g} \mathrm{kg}^{-1}\right)$ & 647 & 644 & 889 & 76,7 & 76,7 & 0,27 & 2,18 & 12 \\
\hline Argila $\left(\mathrm{g} \mathrm{kg}^{-1}\right)$ & 281 & 294 & 396 & 63,3 & 63,3 & $-0,97$ & 1,22 & 22 \\
\hline
\end{tabular}

maior gradiente no solo PVA, típica de solos com horizonte B textural.

Os coeficientes de variação (CV) para P, K, Ca e Mg, são altos; moderados para MO, CTC, V(\%) e argila; e baixos para $\mathrm{pH}$, nas duas áreas; para areia, o $\mathrm{CV}$ foi alto no $\mathrm{LV}$ e baixo no PVA, na profundidade de 0-0,2 m (Tabela 1), de acordo com classificação proposta por Wilding \& Drees (1983). Na profundidade de 0,6-0,8 m, são altos para P, K, $\mathrm{Ca}, \mathrm{Mg}$ e CTC; moderados para $\mathrm{MO}$ e baixo para $\mathrm{pH}$, em ambas as áreas. Alta variabilidade para $\mathrm{P}, \mathrm{pH}$ e V(\%) também foi constatada por Berg \& Klamt (1997b) na camada superficial de um Latossolo Vermelho, com distância entre pontos de 250-300 m.

Os resultados da análise geoestatística encontram-se na Tabela 2. Todos os semivariogramas obtidos apresentaramse bem ajustados, com valores de $\mathrm{R}^{2}$ (coeficiente de determinação) acima de 0,62 e os menores valores de SQR (soma de quadrados reduzida). Para o solo LV (Tabela 2) todas as variáveis analisadas mostraram dependência espacial na profundidade de $0-0,2 \mathrm{~m}$ e somente a CTC na profundidade de 0,6-0,8 m não apresentou dependência espacial. Para o solo PVA (Tabela 2), na profundidade de 0-0,2 m, Ca e argila não indicaram dependência espacial, ocorrendo o mesmo para $\mathrm{P}, \mathrm{MO}, \mathrm{K}, \mathrm{Mg}$, areia e argila na profundidade de 
0,6-0,8 m; isto indica que os intervalos de amostragem utilizados para este solo não foram eficientes para identificar a dependência espacial. Resultados similares foram encontrados por Macedo et al. (1998), segundo os quais solos arenosos apresentam maior variabilidade, sugerindo uma amostragem mais densa quanto às características físicas e químicas que os solos de textura mais argilosa.

O efeito pepita puro, ou seja, semivariograma sem estrutura definida, pode ser justificado pelo fato dessa área estar recebendo adubações dos nutrientes há muito tempo, o que vem corroborar com as afirmações de Cambardella et al.
(1994), de que a dependência espacial fraca ou a ocorrência de estrutura não definida se deve as variações extrínsecas, causadas pela aplicação de fertilizantes, preparo do solo, outras práticas de cultivo e erosões que já ocorreram. Os mesmos autores sugerem que uma variável pode apresentar melhor estrutura espacial com adoção de um espaçamento menor de amostragem, e isto pode ser proposto para trabalhos futuros no solo PVA.

Os modelos ajustados para o solo LV (Tabela 2) foram esféricos para as variáveis MO, Ca, CTC, areia e argila, exponenciais para $\mathrm{P}, \mathrm{pH}, \mathrm{K}, \mathrm{Mg}$, e $\mathrm{V}(\%)$ na profundidade

Tabela 2. Modelo e parâmetros estimados dos semivariogramas ajustados aos dados das variáveis estudadas para os solos Latossolo Vermelho (LV) e Argissolo Vermelho-Amarelo (PVA)

\begin{tabular}{|c|c|c|c|c|c|c|c|}
\hline Variáveis & Modelo & $\mathrm{C}_{0}$ & Patamar & $A_{0}$ & $\mathrm{C}_{0} / \mathrm{C}_{0}+\mathrm{C}_{1}$ & $\mathbf{R}^{2}$ & SQR \\
\hline \multicolumn{8}{|l|}{ SOLO LV $\quad 0-0,2 \mathrm{~m}$} \\
\hline$P\left(m g d m^{-3}\right)$ & Exp & 78,90 & 157,90 & 5106 & 0,500 & 0,814 & 436 \\
\hline $\mathrm{MO}\left(\mathrm{g} \mathrm{dm}^{-3}\right)$ & Esf & 11,29 & 29,13 & 3478 & 0,388 & 0,989 & 3,77 \\
\hline $\mathrm{pH}$ em $\mathrm{CaCl}_{2}$ & Exp & 0,03 & 0,12 & 404 & 0,310 & 0,861 & 0,00014 \\
\hline $\mathrm{K}\left(\mathrm{mmol}_{\mathrm{c}} \mathrm{dm}^{-3}\right)$ & Exp & 2,16 & 4,60 & 1430 & 0,469 & 0,899 & 0,435 \\
\hline $\mathrm{Ca}\left(\mathrm{mmol}_{\mathrm{c}} \mathrm{dm}_{-3}\right)$ & Esf & 122,50 & 265,00 & 8470 & 0,462 & 0,937 & 1413 \\
\hline $\mathrm{Mg}\left(\mathrm{mmol}_{\mathrm{c}} \mathrm{dm}^{-3}\right)$ & Exp & 14,40 & 51,41 & 717 & 0,280 & 0,873 & 77,4 \\
\hline CTC & Esf & 116,20 & 289,40 & 6562 & 0,402 & 0,969 & 705 \\
\hline $\mathrm{V}(\%)$ & Exp & 46,10 & 152,00 & 385 & 0,303 & 0,617 & 788 \\
\hline Areia $\left(\mathrm{g} \mathrm{kg}^{-1}\right)$ & Esf & 2560 & 23980 & 8270 & 0,107 & 0,990 & 5069000 \\
\hline Argila $\left(\mathrm{g} \mathrm{kg}^{-1}\right)$ & Esf & 1680 & 11470 & 10000 & 0,146 & 0,972 & 2276000 \\
\hline \multicolumn{8}{|c|}{ SOLO LV $\quad 0,6-0,8 \mathrm{~m}$} \\
\hline$P\left(\mathrm{mg} \mathrm{dm}^{-3}\right)$ & Exp & 5,19 & 14,11 & 1540 & 0,368 & 0,920 & 6,37 \\
\hline $\mathrm{MO}\left(\mathrm{g} \mathrm{dm}^{-3}\right)$ & Exp & 8,19 & 18,47 & 3420 & 0,443 & 0,956 & 3,42 \\
\hline $\mathrm{pH}$ em $\mathrm{CaCl}_{2}$ & Esf & 0,09 & 0,33 & 5020 & 0,286 & 0,968 & 0,002754 \\
\hline $\mathrm{K}\left(\mathrm{mmol}_{\mathrm{c}} \mathrm{dm}^{-3}\right)$ & Exp & 0,19 & 0,71 & 493 & 0,264 & 0,636 & 0,0351 \\
\hline $\mathrm{Ca}\left(\mathrm{mmol}_{\mathrm{c}} \mathrm{dm}^{-3}\right)$ & Esf & 37,10 & 113,80 & 3520 & 0,326 & 0,949 & 376 \\
\hline $\mathrm{Mg}\left(\mathrm{mmol}_{\mathrm{c}} \mathrm{dm}^{-3}\right)$ & Exp & 3,79 & 12,92 & 900 & 0,293 & 0,767 & 13,8 \\
\hline CTC & EPP & 169,75 & - & - & - & - & - \\
\hline $\mathrm{V}(\%)$ & Esf & 109,30 & 280,70 & 4263 & 0,389 & 0,973 & 865 \\
\hline Areia $\left(\mathrm{g} \mathrm{kg}^{-1}\right)$ & Esf & 2200 & 18340 & 7900 & 0,120 & 0,992 & 2480000 \\
\hline Argila $\left(\mathrm{g} \mathrm{kg}^{-1}\right)$ & Esf & 2150 & 9403 & 11330 & 0,229 & 0,965 & 1267000 \\
\hline \multicolumn{8}{|c|}{ SOLO PVA $\quad 0-0,2 \mathrm{~m}$} \\
\hline$P\left(m g d m^{-3}\right)$ & Esf & 40,30 & 123,70 & 2140 & 0,326 & 0,888 & 590 \\
\hline $\mathrm{MO}\left(\mathrm{g} \mathrm{dm}^{-3}\right)$ & Esf & 6,61 & 13,23 & 1188 & 0,500 & 0,760 & 3,5 \\
\hline $\mathrm{pH}$ em $\mathrm{CaCl}_{2}$ & Exp & 0,11 & 0,38 & 518 & 0,279 & 0,830 & 0,00416 \\
\hline $\mathrm{K}\left(\mathrm{mmol}_{\mathrm{c}} \mathrm{dm}^{-3}\right)$ & Esf & 0,18 & 1,09 & 773 & 0,165 & 0,610 & 0,0713 \\
\hline $\mathrm{Ca}\left(\mathrm{mmol}_{\mathrm{c}} \mathrm{dm}^{-3}\right)$ & EPP & 83,49 & - & - & - & - & - \\
\hline $\mathrm{Mg}\left(\mathrm{mmol}_{\mathrm{c}} \mathrm{dm}^{-3}\right)$ & Exp & 5,51 & 21,12 & 448 & 0,261 & 0,587 & 29,8 \\
\hline CTC & Exp & 127,70 & 255,50 & 4380 & 0,500 & 0,671 & 2165 \\
\hline $\mathrm{V}(\%)$ & Exp & 79,80 & 297,50 & 814 & 0,268 & 0,887 & 2580 \\
\hline Areia $\left(\mathrm{g} \mathrm{kg}^{-1}\right)$ & Esf & 1333 & 2667 & 3560 & 0,500 & 0,871 & 271539 \\
\hline Argila $\left(\mathrm{g} \mathrm{kg}^{-1}\right)$ & EPP & 2376 & - & - & - & - & - \\
\hline \multicolumn{8}{|c|}{ SOLO PVA $\quad 0,6-0,8 \mathrm{~m}$} \\
\hline$P\left(m g d m^{-3}\right)$ & EPP & 0,99 & - & - & - & - & - \\
\hline $\mathrm{MO}\left(\mathrm{g} \mathrm{dm}^{-3}\right)$ & EPP & 3,40 & - & - & - & - & - \\
\hline $\mathrm{pH}$ em $\mathrm{CaCl}_{2}$ & Exp & 0,04 & 0,19 & 392 & 0,22 & 0,65 & 0,00287 \\
\hline $\mathrm{K}$ (mmolc dm-3) & EPP & 0,71 & - & - & - & - & - \\
\hline $\mathrm{Ca}\left(\mathrm{mmol}_{\mathrm{c}} \mathrm{dm}^{-3}\right)$ & Exp & 61,00 & 189,40 & 9110 & 0,32 & 0,63 & 1040 \\
\hline $\mathrm{Mg}\left(\mathrm{mmol}_{\mathrm{c}} \mathrm{dm}^{-3}\right)$ & EPP & 6,11 & - & - & - & - & - \\
\hline CTC & Esf & 188,00 & 872,60 & 7225 & 0,21 & 0,92 & 15514 \\
\hline $\mathrm{V}(\%)$ & Exp & 168,00 & 485,40 & 9110 & 0,34 & 0,70 & 4638 \\
\hline Areia $\left(\mathrm{g} \mathrm{kg}^{-1}\right)$ & EPP & 4522,13 & - & - & - & - & - \\
\hline Argila $\left(\mathrm{g} \mathrm{kg}^{-1}\right)$ & EPP & 3530,74 & - & - & - & - & - \\
\hline
\end{tabular}


de 0-0,2 m, e esféricos para $\mathrm{pH}, \mathrm{Ca}, \mathrm{V}(\%)$, areia e argila, exponenciais para $\mathrm{P}, \mathrm{MO}, \mathrm{K}$ e $\mathrm{Mg}$ na profundidade de 0,6-0,8 m. Para o solo PVA (Tabela 2) os modelos foram esféricos para as variáveis $\mathrm{P}, \mathrm{MO}, \mathrm{K}$ e areia, exponenciais para $\mathrm{pH}, \mathrm{Mg}, \mathrm{CTC}, \mathrm{V}(\%)$, e efeito pepita puro (EPP) para Ca e argila na camada de 0-0,2 m, esférico para CTC e exponenciais para $\mathrm{pH}, \mathrm{Ca}, \mathrm{V}(\%)$ e EPP para as demais variáveis estudadas na camada de $0,6-0,8 \mathrm{~m}$. O modelo esférico é o mais utilizado e adaptado para descrever o comportamento de semivariogramas de propriedades de plantas e de solos (Trangmar et al., 1985; Cambardella et al., 1994; Salviano et al., 1998).

Neste trabalho observou-se que, em geral, as variáveis apresentaram moderado grau de dependência espacial nas duas profundidades para os dois solos estudados, de acordo com os critérios estabelecidos por Cambardella et al. (1994). Resultados semelhantes foram obtidos por Pocay (2000), Souza (2001) e Araújo (2002) em que a maioria das variáveis químicas e granulométricas apresentou grau moderado de dependência espacial; entretanto, observaram-se valores pouco mais elevados da relação $\mathrm{C}_{0} /\left(\mathrm{C}_{0}+\mathrm{C}_{1}\right)$ na profundidade de $0-0,2 \mathrm{~m}$ para ambos os solos, indicando que os solos podem apresentar variabilidade em escalas menores que a utilizada neste estudo. As variáveis que mostraram forte dependência espacial foram as granulométricas do solo LV, nas duas profundidades; $K$, na profundidade de $0-0,2 \mathrm{~m}$ e CTC, na profundidade de 0,6-0,8 m, do solo PVA. Segundo Cambardella et al. (1994), a forte dependência espacial das variáveis, como textura e mineralogia, está associada a variações intrínsecas de propriedades do solo.

Os mapas tridimensionais gerados pela krigagem do solo LV se acham nas Figuras 2 a 4, para o solo PVA e nas Figuras 5 a 7 .

Analisando-se a Figura 2 (A, B, C e D), nota-se que a areia está em maiores teores na camada superficial e, em contrapartida, a argila é maior na profundidade de 0,60,8 m, mas onde o solo é mais argiloso na superfície, esta característica permanece nas camadas mais profundas, devido ao movimento de água ao longo do perfil do solo, que carrega as partículas mais finas para camadas mais profundas. Pode-se observar que, para a areia e argila, existe uma continuidade espacial maior na direção $135^{\circ}$, a qual desaparece em sua extremidade, próximo às margens do rio Mogi-Guaçu. Pode-se, também, atribuir este comportamento ao fluxo superficial da água nas encostas, que carrega as partículas de areia para os locais de baixa altitude, devido ao transporte de partículas pela erosão entre sulcos, indicando que a distribuição das partículas do solo obedece principalmente aos fatores de formação e evolução do solo. Outra hipótese que pode ser levantada é que nesses locais onde o teor de areia é mais alto, foi verificada a ocorrência de basalto amigdaloidal, que possui, em sua composição mineralógica, quartzo, que pode estar contribuindo com partículas da fração areia.

Para as variáveis químicas do solo LV observa-se que os teores na profundidade de $0-0,2$ m são maiores que na profundidade de 0,6-0,8 m (Figuras 3 e 4).
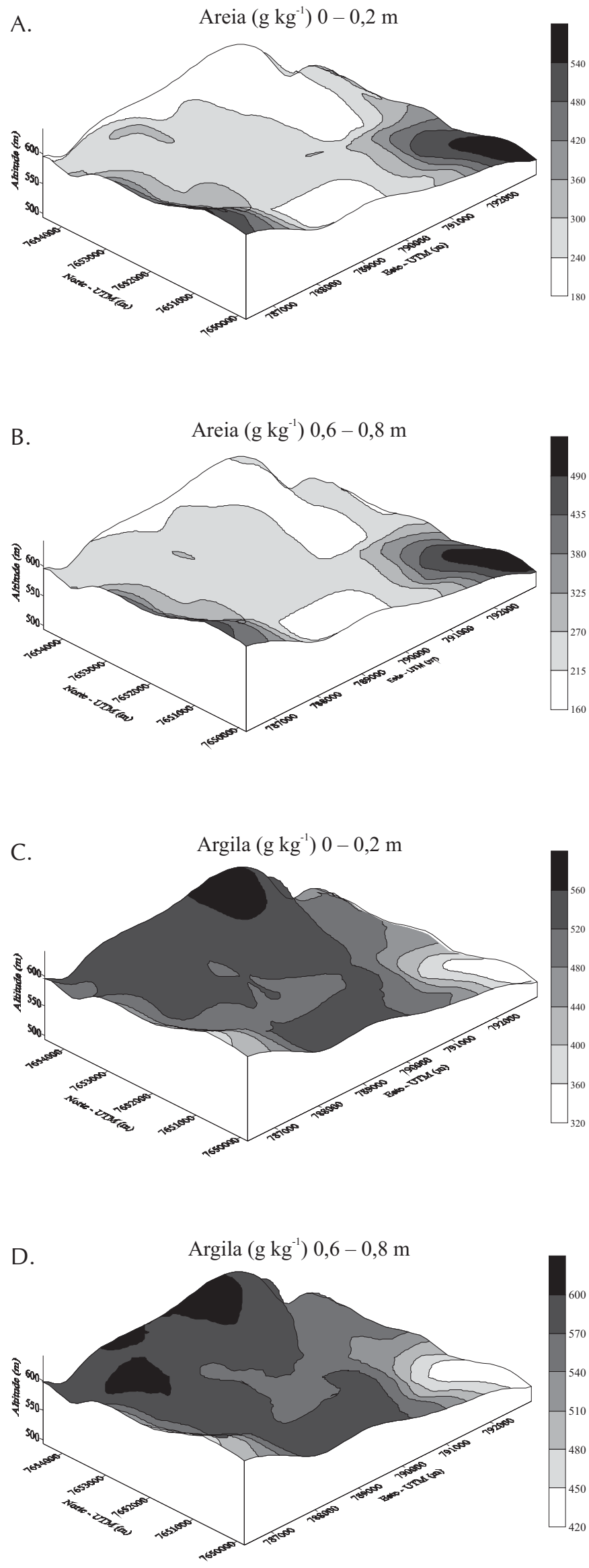

Figura 2. Mapas tridimensionais das variáveis areia e argila da área do solo Latossolo Vermelho (LV), nas duas profundidades 

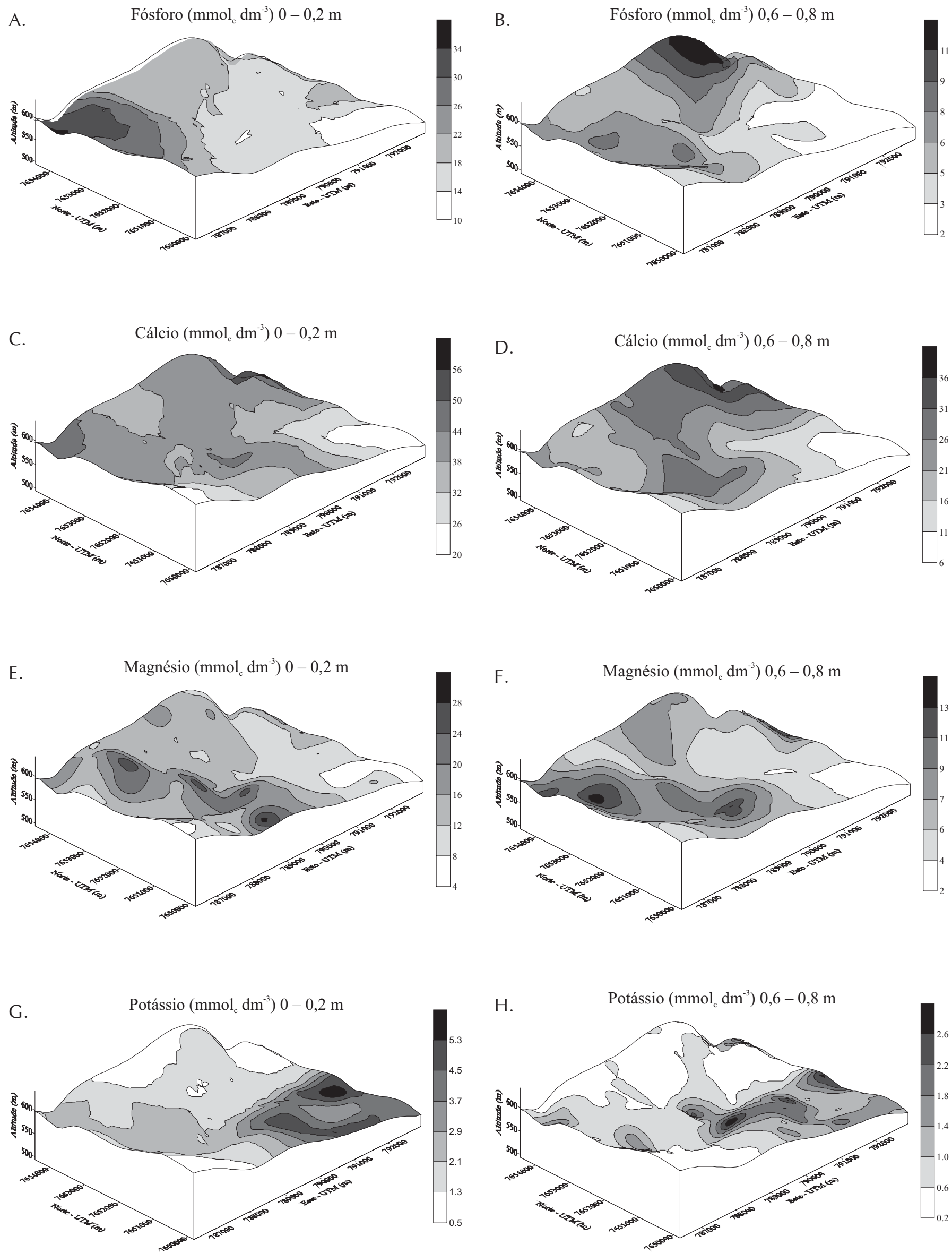

Figura 3. Mapas tridimensionais das variáveis Fósforo (P), Cálcio (Ca), Magnésio (Mg) e Potássio (K) da área do solo Latossolo Vermelho (LV), em duas profundidades

R. Bras. Eng. Agríc. Ambiental, v.10, n.2, p.294-305, 2006. 

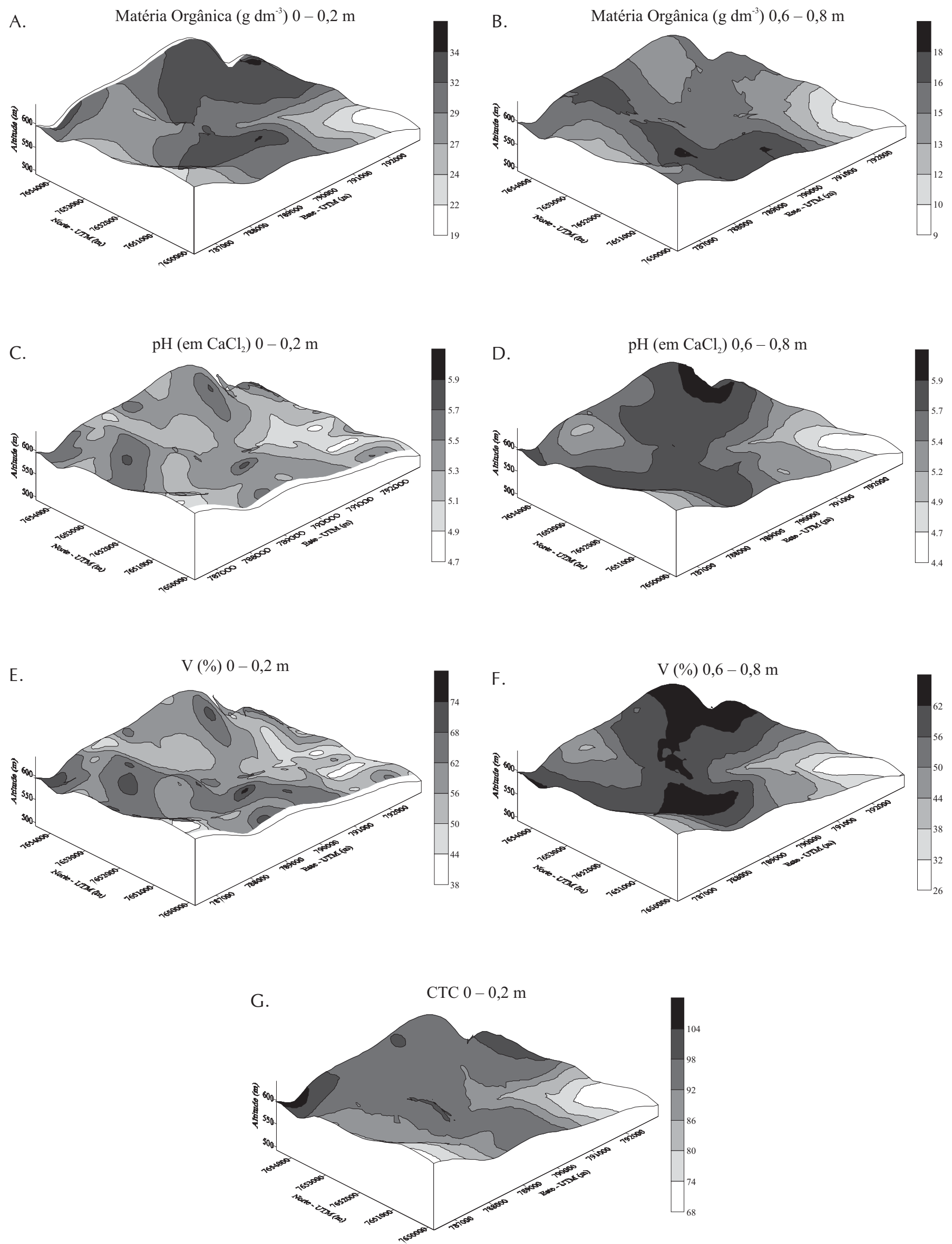

Figura 4. Mapas tridimensionais das variáveis Matéria Orgânica (MO), pH, V (\%) e CTC da área do solo Latossolo Vermelho (LV), em duas profundidades 

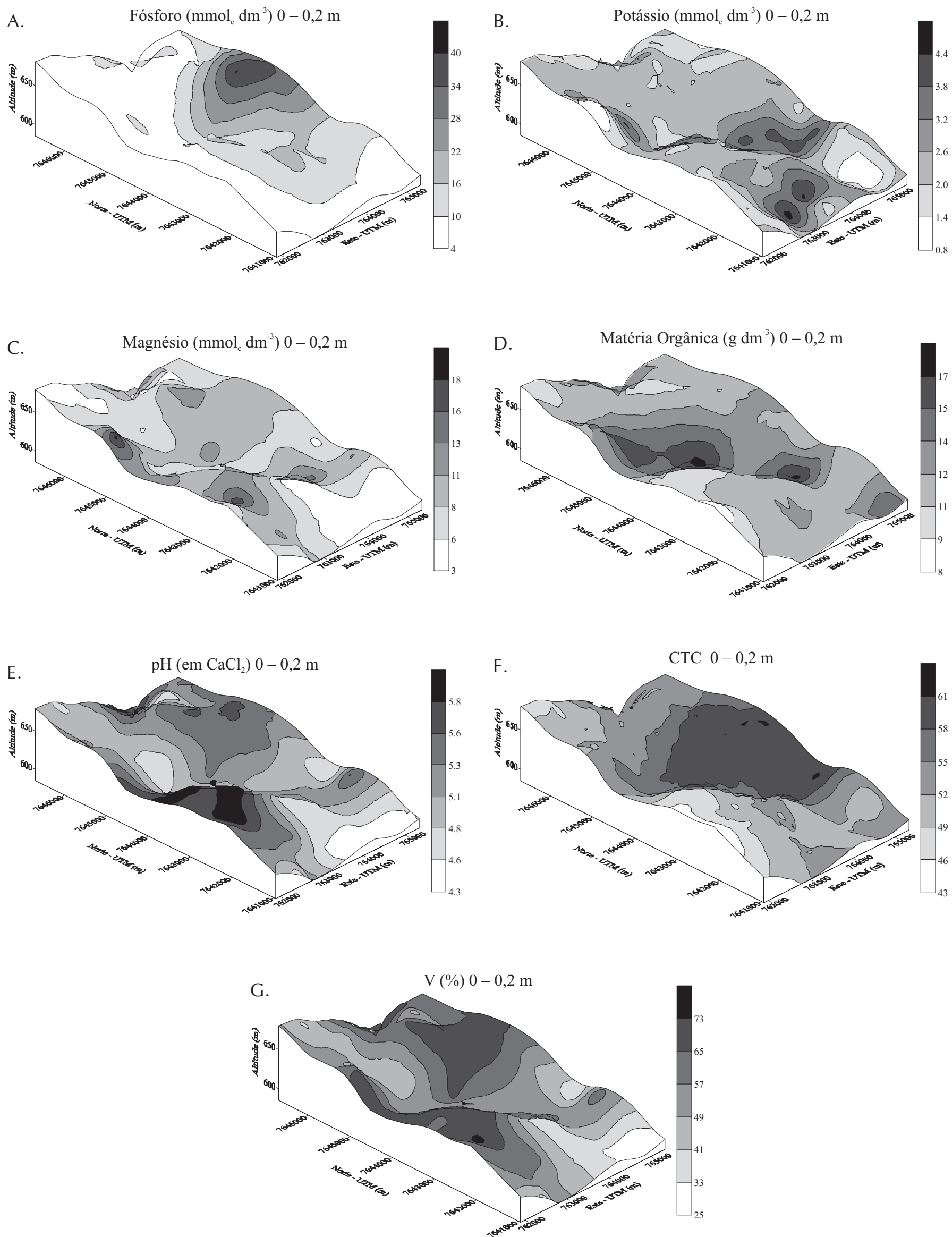

Figura 5. Mapas tridimensionais das variáveis Fósforo (P), Potássio (K), Magnésio (Mg), Matéria Orgânica (MO), pH, CTC e V (\%) da área do solo Argissolo Vermelho-Amarelo (PVA), na profundidade $0-0,2 \mathrm{~m}$ 
O fósforo (P) apresenta distribuição espacial diferenciada das outras bases do solo, com teores mais baixos em altitudes menores, e é possível observar que na profundidade de 0,6-0,8 m os maiores teores coincidem com os locais de maiores teores de argila, o que se explica pelo fato de que nas partes com menores altitudes o solo tem cor mais amarela (2,5 YR 2,5/4), podendo indicar um incremento da goethita, o qual apresenta maior capacidade de retenção de $\mathrm{P}$ que a hematita, que caracteriza cor mais vermelha do solo, com cor 10R 3/4, nas partes de maiores altitudes. Essas áreas coincidem com maiores teores de $\mathrm{P}$ disponível no solo (Figura 3A e B).

O potássio (K) mostra comportamento diferente e maiores teores nas áreas com menores altitudes, na profundidade de $0-0,2 \mathrm{~m}$ (Figura 3G). Um dos fatores que podem estar influenciando, é a alta mobilidade desse nutriente no solo, ocorrendo sua deposição nas partes baixas do terreno; outra possível explicação refere-se ao fato de que este nutriente possa estar disponibilizado pelo intemperismo de minerais primários, provavelmente feldspato potássico, presentes na fração areia do solo LV.

A fração areia do solo LV (Figura 2A e B) apresenta-se em altos teores nos locais onde o potássio é encontrado em maior quantidade, e isto ocorre nas duas profundidades estudadas, pelo fato do solo LV se localizar em uma região de transição entre basaltos e arenitos onde, provavelmente, pode ser encontrado o feldspato potássico.
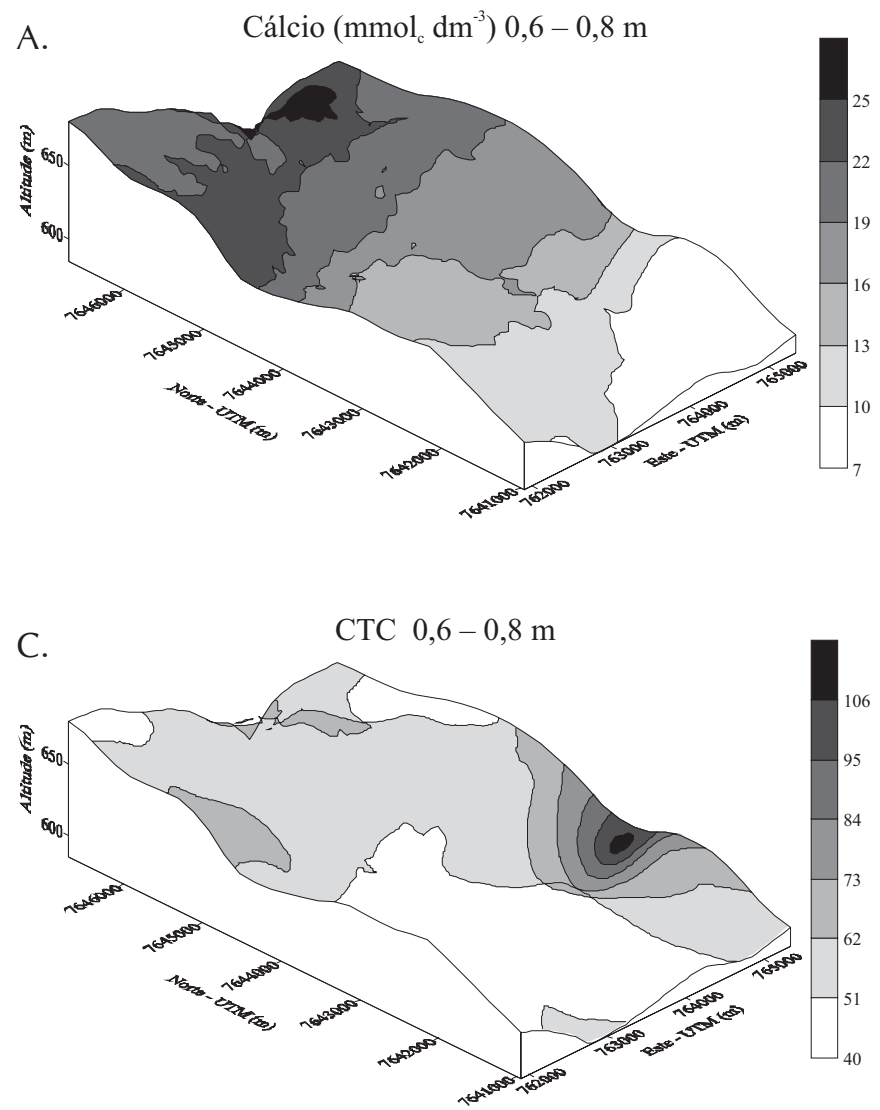

O cálcio e o magnésio apresentam distribuições espaciais mais descontínuas e diferentes entre camadas para o solo LV nas duas profundidades, ocorrendo os maiores teores nas áreas de maior altitude, onde o solo é mais plano e a lixiviação de bases reduzida, em virtude desses nutrientes serem aplicados em maiores quantidades, em relação aos outros, por meio da calagem realizada pelos produtores rurais (Figura 3 (E, F, G e H).

Observa-se que o valor médio do teor de MO do solo LV (Figura 4A e B) na camada de 0-0,2 m, é bem maior que o encontrado para o solo PVA (Figura 5D). Nos locais em que os teores de MO são maiores, o solo é mais argiloso, visto que solos com textura argilosa como o LV, armazenam melhor a MO, e esta pode estar contribuindo para os maiores valores de CTC, devido ao desenvolvimento de cargas.

A camada de 0-0,2 m é mais afetada pelo processo de mecanização e erosão, o que está, possivelmente, influenciando na distribuição das propriedades do solo, em diferentes posições do terreno, em razão do arrastamento de partículas do solo, que podem estar ocasionando comportamento espacial descontínuo nos teores das propriedades do solo analisadas.

$\mathrm{O}$ solo LV tem maior variabilidade espacial de $\mathrm{pH}$ na profundidade de $0-0,2 \mathrm{~m}$ que na profundidade de $0,6-$ $0,8 \mathrm{~m}$; este mesmo resultado foi encontrado por Berg \& Klamt (1997a) que concluíram que a maior variância para pH a curtas distâncias $(50 \mathrm{~m})$ mostrou-se relacionada à
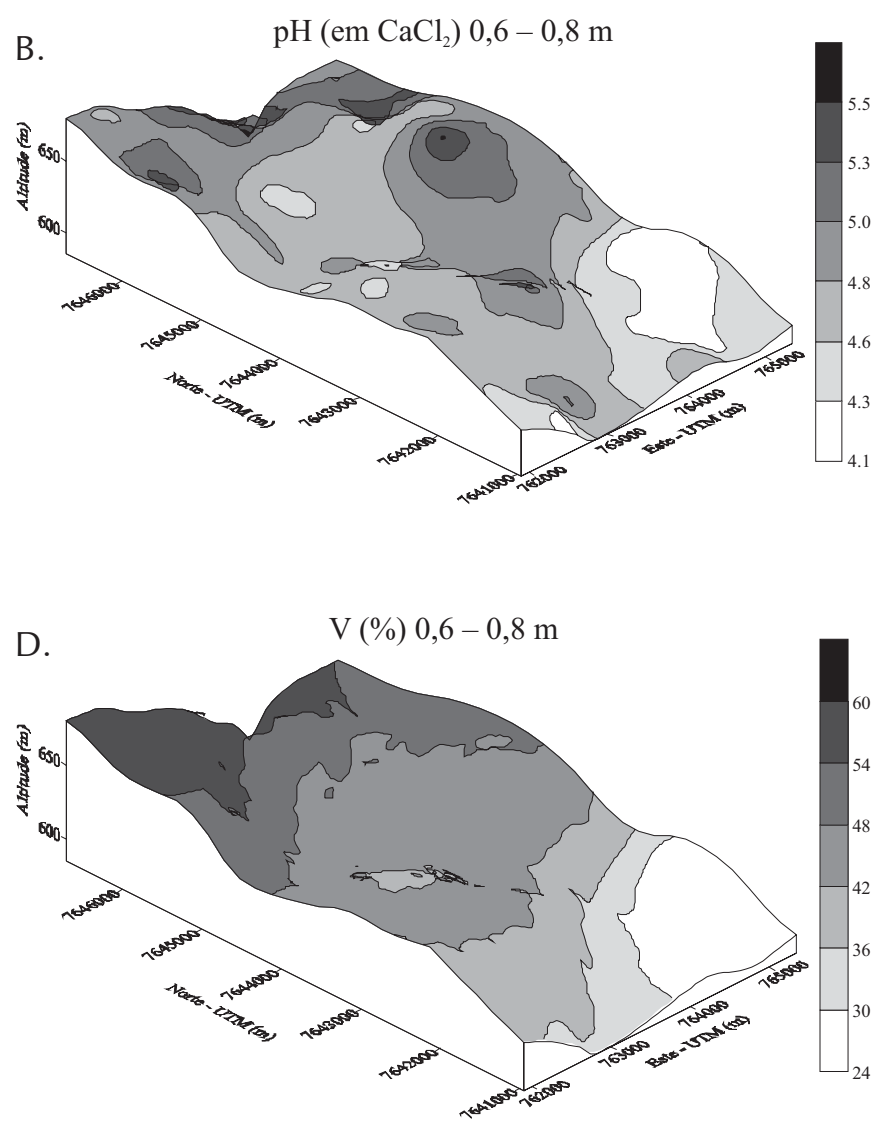

Figura 6. Mapas tridimensionais das variáveis Cálcio (Ca), pH, CTC e V (\%) da área do solo Argissolo Vermelho-Amarelo (PVA) na prlofundidade 0,6 - 0,8 m 


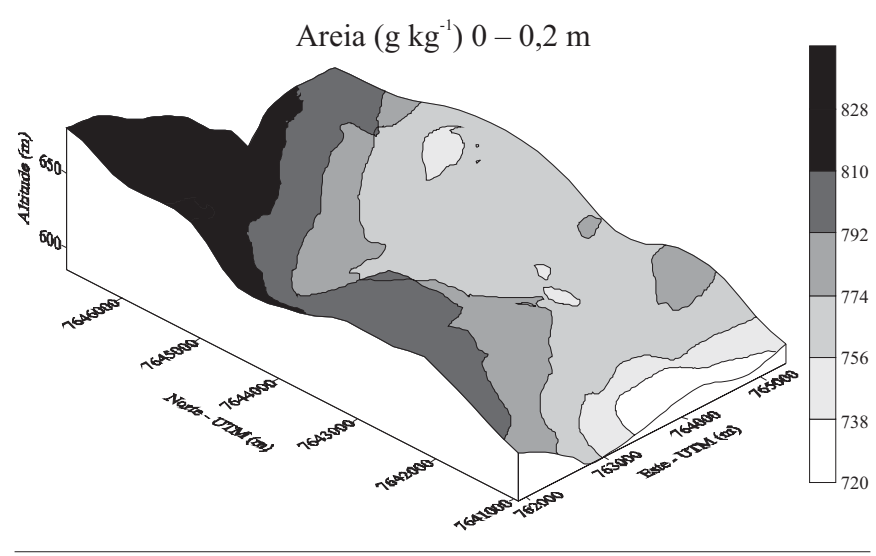

Figura 7. Mapa tridimensional da variável areia da área do solo Argissolo Vermelho-Amarelo (PVA), na profundidade $0-0,2 \mathrm{~m}$

distribuição não homogênea de calcário e à grande variância entre glebas, na quantidade de corretivo utilizada pelos produtores. Em relação à CTC e ao V(\%) deste mesmo solo, estas apresentam menor continuidade espacial, provavelmente por serem dependentes dos teores de $\mathrm{MO}$ e de bases ocorrendo, com isso, associação da variabilidade espacial do $\mathrm{K}, \mathrm{Ca}, \mathrm{Mg}$ e $\mathrm{H}+\mathrm{Al}$. Os maiores valores de CTC do solo LV ocorrem nos locais em que os teores de $\mathrm{MO}$ e argila são maiores, porque estas contribuem para o maior desenvolvimento de cargas negativas no solo e, conseqüentemente, maior armazenamento de nutrientes. O mesmo comportamento é observado no solo PVA, sendo a CTC e o V(\%) mais baixos, em virtude do maior conteúdo de areia (Figura 5F e G e Figura 6C e D). Observa-se, também maior continuidade espacial para Ca e $\mathrm{V}(\%)$ no sentido Este no solo PVA, sobretudo nas encostas, onde o solo é mais raso e está próximo do material de origem, que é um arenito com cimento calcário o qual, durante o intemperismo, libera Ca para o solo, aumentando a saturação por bases.

No geral, as variáveis químicas do solo PVA apresentaram comportamento bastante semelhante ao do solo LV, mas é conveniente observar que os níveis dos nutrientes no solo PVA são baixos em comparação com os do solo LV, devido à menor capacidade dos solos arenosos em armazenarem os nutrientes.

Em relação às variáveis físicas do solo PVA (Figura 7), somente a areia apresentou dependência espacial, ocorrendo os maiores teores de areia nas maiores altitudes, que apresentam declive mais acentuado, o solo menos intemperizado e material de origem mais próximo da superfície.

A variabilidade espacial do solo PVA é bem mais acentuada e condicionada pelo relevo ondulado ocorrendo, então, superfícies com comportamento diferenciado, além de erosões em sulcos que progridem para voçorocas em alguns locais, devido à não realização do controle conservacionista, como se observa no campo. Salviano et al. (1998) afirmaram que a erosão acelerada do solo é um fator que pode contribuir para aumentar a variabilidade espacial, ou seja, erosões pretéritas podem ter influenciado na atual variabilidade espacial deste solo.

\section{CONCLUSÕES}

1. As propriedades do solo estudadas apresentaram dependência espacial, com exceção da CTC na profundidade de 0,6-0,8 m, para o solo LV, e Ca e argila na profundidade de 0-0,2 m, e P, MO, K, Mg, areia e argila na camada de 0,6-0,8 m no solo PVA.

2. O solo PVA apresenta menor continuidade espacial, principalmente na camada de 0,6-0,8 m.

3. O solo LV apresenta zonas mais homogêneas de fertilidade na profundidade de $0-0,2 \mathrm{~m}$, sendo o manejo deste solo facilitado.

\section{LITERATURA CITADA}

Araujo, A. V. Variabilidade espacial de propriedades químicas e granulométricas do solo na definição de zonas homogêneas de manejo. Jaboticabal: FCAV/UNESP, 2002. 80p. Dissertação Mestrado

Beckett, P. H. T.; Webster, R. Soil variability: a review. Soils and Fertilizers, Farnham Royal, v.34, p.1-15,1971.

Berg, M. V. d; Klamt, E. Variabilidade espacial de características de solos na região do Planalto Médio, RS: I. Análise da variância por amostragem aninhada. Revista Brasileira de Ciência do Solo, Viçosa, v.21, n.2, p.393-399, 1997a.

Berg, M. V. de; Klamt, E. Variabilidade espacial de características de solos na região do Planalto Médio, RS: II. Análise da semivariância e da variância. Revista Brasileira de Ciência do Solo, Viçosa, v.21, n.2, p.401-408, 1997b.

Burrough, P. A.; Varekamp, C.; Skidmore, A. K. Using public domain geostatistical and GIS software for spatial interpolation. Photogrammetric Engineering \& Remote Sensing, Maryland, v.62, n.7, p.845-854, 1996.

Cambardella, C. A.; Moorman, T. B.; Novak, J. M.; Parkin, T. B.; Karlen, D. L.; Turco, R. F.; Konopka, A. E. Field-scale variability of soil properties in central Iowa soils. Soil Science Society America Journal, Madison, v.58, n. 4, p.15011511, 1994.

Day, P. R. Particle fraction and particle fractination and particlesize analysis. In: Black, C. A. Methods of soil analysis. Madison, American Agronomy, v.1, p.545-566, 1965.

Golden Software. Surfer for windows: realease 6.01. Surface mapping system. Golden, CO, 1995. Programa computacional

Macedo, J. R.; Otonni Filho, T. B.; Meneguelli, N. A. Variabilidade de características físicas, químicas e físico-hídricas em solo Podzólico Vermelho Amarelo de Seropédica, RJ. Pesquisa Agropecuária Brasileira, Brasília, v.33, n.4, p.2043-2053, 1998.

Oliveira, J. J.; Chaves, L. H. G.; Queiroz J. E.; Luna, J. G. Variabilidade espacial de propriedades químicas em um solo salinosódico. Revista Brasileira de Ciência do Solo, Viçosa, v.23, n.3, p.783-789, 1999.

Ovalles, F.; Rey, J. Variabilidad interna de unidades de fertilidad en suelos de la depresión del Lago de Valencia. Agronomia Tropicale, Maracay, v.44, n.1, p.41-65, 1994. 
Pocay, V. G. Relações entre pedoforma e variabilidade espacial de atributos de Latossolos sob cultivo de cana-de-açúcar. Jaboticabal: FCAV/UNESP, 2000. 177p. Dissertação Mestrado

Robertson, G. P. Geostatistics for the environmental sciences $\left(\mathrm{GS}^{+}\right)$versão 5.03 Beta. Plainwell: Gamma Design Software. 1998. 152p. Programa Computacional

Salviano, A. A. C.; Vieira, S. R.; Sparovek, G. Variabilidade Espacial de atributos de solo e de Crotalaria juncea L. em área severamente erodida. Revista Brasileira de Ciência do Solo, Viçosa, v.22, n.1, p.115-122, 1998.

Schlotzhauer, S. D.; Littell, R. C. SAS System for elementary statistical analysis. 2 ed. Cary: SAS Institute Inc, 1997. 456p.

Souza, C. K. Relação solo-paisagem-erosão e variabilidade espacial de Latossolos em áreas sob cultivo de cana-de-açúcar no município de Jaboticabal, SP. Jaboticabal: FCAV/UNESP, 2001. 186p. Dissertação Mestrado

Souza, L. Variabilidade espacial do solo em sistemas de manejo. Porto Alegre: UFRS, 1992. 162p. Tese Doutorado
Souza, L. S.; Cogo, N. P.; Vieira, S. R. Variabilidade de propriedades físicas e químicas do solo em um pomar cítrico. Revista Brasileira de Ciência do Solo, Viçosa, v.21, n.3, p.367-372, 1997.

Trangmar, B. B.; Yost, R. S.; Uehara, G. Application of geoestatistics to spatial studies of soil properties. Advance Agronomy, Orlando, v.38, n.1, p.45-93, 1985.

van Raij, B.; Quaggio, J. A.; Cantarella, H.; Ferreira, M. E.; Lopes, A.S.; Bataglia, O. A. Análise química do solo para fins de fertilidade. Campinas: Fundação Cargill, 1987. $170 \mathrm{p}$.

Vieira, S. R. Geoestatística em estudos de variabilidade espacial do solo. In: Novais, R. F.; Alvares, V. H.; Schaeffer, C. E. G. R. Tópicos em ciência do solo, Viçosa: Sociedade Brasileira de Ciência do Solo, 2000. p.1-54.

Wilding, L. P.; Drees, L. R. Spatial variability and pedology. In: Pedogenesis and soil taxonomy: concepts and interactions. New York: Elsevier, 1983. p.83-116. 